\title{
NÍVEL DE RUÍDO EM SALA DE PARTO
}

\author{
Fernanda Lima de Campos Oliveiraํㅜ, Tereza Yoshiko Kakehashi², Miriam Harumi Tsunemi , Eliana Moreira \\ Pinheiro ${ }^{4}$
}

\footnotetext{
${ }^{1}$ Aluna da Escola Paulista de Enfermagem (EPE) da Universidade Federal de São Paulo. São Paulo (UNIFESP). Bolsista de Iniciação Científica da Fundação de Amparo à Pesquisa do Estado de São Paulo. São Paulo, Brasil. E-mail: lima@gmail.com.br

${ }^{2}$ Doutora em Enfermagem. Professora da Escola de Terapias Orientais de São Paulo. São Paulo, Brasil. E-mail: terezayk@ ig.com.br

${ }^{3}$ Doutora em Estatística. Consultora de estatística do Instituto de Radiologia do Hospital das Clínicas de São Paulo. São Paulo, Brasil. E-mail: miharumi@ig.com.br

${ }^{4}$ Doutora em Enfermagem. Professora Adjunto da EPE/UNIFESP. São Paulo, Brasil. E-mail: elianapinheiro@hotmail.com
}

\begin{abstract}
RESUMO: Teve como objetivo identificar níveis de pressão sonora em salas de parto e fontes de ruído. Estudo quantitativo, descritivo, exploratório. Coleta de dados ocorreu de outubro de 2008 a julho de 2009. Utilizou-se um dosímetro para registrar os níveis de pressão sonora e a observação não-estruturada para identificar fontes geradoras. Realizaram-se 2.928 minutos de registros do ruído e observação de fontes. Valores de nível de pressão sonora equivalente (Leq) foram calculados pelo software Quest Suit. Identificou-se Leq médio de 69,6 dBA $( \pm 3,69)$ em partos normais e 65,4 dBA $( \pm 2,28)$ em cesáreas. Fonte mais frequente de ruído foi diálogo entre profissionais. Os níveis de pressão sonora estão acima dos recomendados pelos órgãos competentes e a principal fonte de ruído está relacionada ao comportamento dos profissionais. Faz-se necessário implementar programas educativos a fim de amenizar o ruído e melhorar a assistência ao neonato e família e as condições de trabalho.
\end{abstract}

DESCRITORES: Ruído. Parto humanizado. Recém-nascido.

\section{NOISE LEVELS IN THE DELIVERY ROOM}

\begin{abstract}
This quantitative, descriptive, and exploratory study aimed to identify sound pressure levels and their sources in university hospital delivery rooms in Brazil. Data was collected between October, 2008, and July, 2009. A dosimeter was used to register noise and non-structured observation was used to identify noise sources. Noise and source observations were registered over 2928 minutes. Mean equivalent sound levels (Leq) were measured using Quest Suit software. Mean Leq values were identified as 69.6 dBA $( \pm 3.69)$ during vaginal deliveries and $65.4 \mathrm{dBA}( \pm 2.28)$ during cesarean deliveries. The most frequent noise source was conversation among professionals in the room. Considering the high levels of sound pressure and the sources, this study points out the need for intervention through educational programs among professionals and students to minimize noise and improve the care provided to the newborn and his/her family, as well as professional working conditions.
\end{abstract}

DESCRIPTORS: Noise. Humanized delivery. Newborn.

\section{NIVEL DE RUIDO EN LA SALA DE PARTO}

RESUMEN: El presente estudio tuvo por objetivo identificar los niveles de presión acústica en salas de parto y las fuentes que producen ruido. Se trata de un estudio cuantitativo, descriptivo, exploratorio. La recolección de los datos se hizo de octubre de 2008 a julio de 2009. Se utilizó un decibelímetro para registrar los niveles de presión acústica y la observación no estructurada para identificar las fuentes generadoras de ruido. Se realizaron 2928 minutos de registros de ruido y observación. Los valores de niveles de presión acústica equivalentes (Leq), fueron calculados por el software Quest Suit. Se identificó Leq promedio de 69,6 dBA $( \pm 3,69)$ en partos normales y 65,4 dBA $( \pm 2,28)$ en cesáreas. El diálogo entre los profesionales fue la fuente más frecuente de ruido. Los niveles de presión sonora registrados son superiores a los recomendados por organismos especializados, y la principal fuente de ruido se relaciona con la conducta de los profesionales. Es necesario implementar programas educativos con el fin de minimizar el ruido y mejorar la atención del recién nacido, de su familia y las condiciones de trabajo.

DESCRIPTORES: Ruido. Parto humanizado. Recién nacido. 


\section{INTRODUÇÃO}

Observa-se que pouca atenção é dada à ecologia ambiental na assistência de enfermagem ao Recém-Nascido (RN), parturiente e família em sala de parto. Essa temática torna-se cada vez mais importante, uma vez que os avanços técnico-científicos observados nos últimos anos modificaram profundamente o ambiente de cuidado ao recém-nascido e família, constituindo hoje, um dos focos de atenção dos profissionais que os assistem. O manejo do som ambiental constitui-se em um importante fator para o conforto da clientela e segurança para o desempenho das atividades dos profissionais.

O som é uma forma de energia que é gerada pela colisão de moléculas do meio, produzindo variações de pressão e, por serem ondas mecânicas, necessitam de meio físico, por exemplo, o ar, para sua propagação. ${ }^{1} \mathrm{O}$ ruído é um tipo de som desorganizado, em frequências fisiologicamente incompatíveis com o ouvido humano, que podem produzir lesões físicas, alterações psíquicas e comportamentais, ${ }^{2-3}$ cuja característica está associada ao conceito de som indesejado e desagradável.

As diversas fontes que produzem ruído em salas de parto, algumas vezes, parecem atingir Níveis de Pressão Sonora (NPSs) tão elevados, que podem ser incompatíveis para um momento tão singular na vida da família, que é o nascimento do bebê. A legislação brasileira preconiza NPS para algumas unidades hospitalares em geral, como apartamentos, enfermarias, berçários e centro cirúrgico, recomendando que esses permaneçam entre 35 e $45 \mathrm{~dB}^{*}$, sendo o menor valor considerado desejável e o maior o aceitável. ${ }^{4} \mathrm{~A}$ Organização Mundial da Saúde preconiza nível de pressão sonora equivalente (Leq), que representa a média da energia sonora durante um intervalo de tempo, de $45 \mathrm{~dB}$ para área interna hospitalar. ${ }^{5}$ Não existem normas brasileiras recomendando NPS para sala de parto.

Os valores descritos visam o conforto acústico dos pacientes. Para a saúde do trabalhador, o Ministério da Saúde (MS) do Brasil determina que a máxima exposição ao ruído seja de $85 \mathrm{dBA}$, para o turno de trabalho diário de oito horas. ${ }^{6}$

O programa de humanização de parto e nascimento preconizado pelo MS do Brasil recomenda que o ambiente da sala de parto seja acolhedor e silencioso, para que a família possa vivenciar a experiência de nascimento de um novo membro. ${ }^{7}$
Evidenciou-se na literatura nacional e internacional que há escassez de estudos sobre os NPSs em sala de parto; temática ainda muito pouco explorada pelos pesquisadores, quando comparada às pesquisas que identificam os níveis de ruído em unidades de terapia intensiva neonatal e no interior das incubadoras. ${ }^{8-9}$

Partindo da realidade do centro obstétrico de um hospital universitário de referência para o MS na assistência às gestantes e recém-nascidos de alto risco, e que durante a realização dos procedimentos, há um grande número de alunos dentro das salas de parto, questiona-se a que NPSs estão expostos o neonato, a família e os profissionais e quais são as fontes que produzem ruído.

\section{OBJETIVOS}

Essa pesquisa teve por objetivos avaliar o NPS nas salas de parto de um hospital universitário e identificar as fontes que os produziram, desde o momento em que a parturiente é colocada na mesa de parto, até a sua saída da sala.

\section{MÉTODO}

Trata-se de um estudo quantitativo, descritivo e exploratório. ${ }^{10}$ Foi realizado em duas salas do centro obstétrico de um hospital universitário do município de São Paulo - Brasil. As salas de parto possuem área de $13,63 \mathrm{~m}^{2}$ e de $20,00 \mathrm{~m}^{2}$, pé direito de $2,74 \mathrm{~m}$, piso de granito, paredes de fórmica e teto de concreto, ar condicionado, mesa de parto, carrinho de anestesia, berço aquecido e um foco central. Os turnos de trabalho do centro obstétrico são divididos em três plantões: 7 às 13h, 13 às $19 \mathrm{~h}$ e das 19 às 7h.

No referido hospital, ocorrem em média 68 partos mensalmente. De maneira geral, o tempo de duração dos procedimentos de partos normais é de 90 minutos, e das cesáreas, 150 minutos, quando não há intercorrências.

O instrumento utilizado para o registro dos NPSs foi um dosímetro de marca Quest, modelo Q-400, previamente calibrado pelo INMETRO. O dosímetro foi envolvido em espuma e posicionado ao nível da orelha da mãe, por meio de um suporte de soro fixado ao chão. $\mathrm{O}$ aparelho foi mantido a $1,00 \mathrm{~m}$ do piso e a uma distância de aproximadamente $1,60 \mathrm{~m}$ da parede. ${ }^{11}$ Foi programado para registrar NPS entre 40 a $140 \mathrm{~dB}$.

* dB é a décima parte do bel; unidade que mede a intensidade do som. 
O aparelho mede os NPSs em dB e é composto por circuitos eletrônicos e funciona simultaneamente como um cronômetro, calculadora e banco de dados. Funciona por meio de uma bateria que dura aproximadamente 45 horas. ${ }^{12}$ Assim, cada nível de ruído medido teve o tempo de duração precisamente registrado e armazenado, fornecendo um conjunto de dados para tratamento estatístico. Por medida de segurança, antes de iniciar cada coleta, foi trocada a bateria do dosímetro e, também, calibrado o aparelho com um calibrador QC10.

O aparelho foi programado para operar na escala A, em velocidade Fast. Esta velocidade possibilita medir ruídos que não oscilam muito rapidamente. A escala A é a mais frequentemente usada em estudos desta natureza e sua unidade de medida é o dBA, que representa o método de filtragem que mimetiza as características receptivas da orelha humana, sendo indicada pela apreensão de ruídos contínuos (Leq). O Leq é um dado importante para análise dos resultados desta pesquisa, uma vez que o mesmo possibilita conhecer o nível sonoro médio integrado, durante um determinado período de tempo, sabendo-se que lesões à audição humana são provocadas não só por exposição a ruídos elevados, mas também, pela duração dos mesmos. ${ }^{13}$

Simultaneamente, realizou-se a observação não-estruturada. ${ }^{14}$ Nesse processo, o pesquisador registrou em um diário de campo os horários e os eventos que produziam ruído.

Os dados foram coletados no período de outubro de 2008 a julho de 2009, nos plantões da manhã, tarde e noite, de terça a domingo, conforme a disponibilidade da investigadora, após autorização do Comitê de Ética em Pesquisa (processo $\left.n^{\circ} 1026 / 08\right)$. Ressalta-se que nesse parecer foi autorizada a coleta de dados sem que se esclarecessem os objetivos do estudo para a equipe de saúde do serviço. Considerou-se como início do parto, o momento em que a parturiente era colocada sobre a mesa. Nessa ocasião, a pesquisadora, ligava o dosímetro e iniciava também os registros das fontes que produzissem ruído. Ambos os registros eram finalizados quando a puérpera deixava a sala. Durante todo o período de coleta de dados, não houve situação de ocorrer dois partos simultaneamente.

Foram realizadas 48 horas e 48 minutos de registros dos NPSs e de fontes de ruído, sendo cinco horas e 25 minutos correspondentes a cinco partos normais e 43 horas e 23 minutos equivalentes a 21 cesáreas. Os registros de NPS entre os partos normais e cesáreas foram obtidos conforme a ocorrência desses procedimentos. Foram observados mais partos cesáreos, em virtude do referido serviço assistir gestantes de alto risco. Obteve-se o total de 2.928 registros de NPS pelo dosímetro. Os dados do dosímetro foram transferidos para o programa Quest Suit for Excel. Os valores médios dos NPSs foram analisados segundo tipo de parto, distintos plantões, número de pessoas presentes no ambiente e a presença do recém-nascido na sala de parto.

Em virtude da ocorrência maior de partos cesárea do que normais e os NPSs não apresentarem distribuição normal foi utilizado o teste de Mann-Whitnney. O teste ANOVA foi usado para comparar os NPSs dos partos que ocorreram nos diferentes turnos (manhã, tarde e noite), considerando-se que não há relação dos valores registrados entre estes grupos. A comparação dos NPS entre os momentos de permanência do RN na sala de parto (antes, durante e depois) foi realizada através do teste Friedmann, pois as medidas referem-se ao mesmo parto, em momentos distintos. Considera-se uma dependência entre esses momentos, pois os registros de NPS são do mesmo parto.

Empregou-se o teste de Spearmann em virtude do pequeno número de partos observados. O nível descritivo de significância utilizado em todos os testes citados foi 0,05 .

\section{RESULTADOS}

Dos 2.928 minutos de registros dos níveis de pressão sonora obtidos, 968 minutos ocorreram no plantão da manhã (um parto normal e sete cesáreas), 1167 minutos, da tarde (quatro partos normais e sete cesáreas), e 778 minutos, nos plantões noturnos (sete partos cesáreas), perfazendo o total de 26 partos.

Desses registros, 1136 foram obtidos antes dos nascimentos dos bebês, 363 minutos durante a permanência dos recém-nascidos na sala, e 1429 minutos, após a sua saída. O tempo de permanência do neonato no interior da sala variou de sete a 21 minutos e o da parturiente foi de 28 a 120 minutos nos partos normais e de 87 a 187 minutos, nas cesáreas.

Quanto ao tipo de parto, observou-se que os Leq médios entre todos os partos normais e cesáreas foram de 69,6 6 3,69 dBA e de 65,4 4 2,28 $\mathrm{dBA}$, respectivamente. A variação do Leq entre todos os partos estudados foi de $4,2 \mathrm{dBA}$. 
Pelo teste de Mann-Whitnney concluiuse que existe uma diferença estatisticamente significativa entre os NPS desses dois tipos de partos ( $p$-valor $=0,008$ ), ou seja, os partos normais apresentam Leq significativamente maior que os partos cesáreas monitorados. Comparando os Leq médios por plantões e entre todos os partos normais e cesáreas pesquisados, identificou-se, quantitativamente, que o maior Leq foi de 67,4 dBA, com desvio-padrão igual a 2,33 dBA durante o período noturno. Por outro lado, o menor Leq médio registrado foi no período da manhã, igual a 65 dBA, com desvio-padrão de 1,44 dBA.
A tabela 1 apresenta as médias e os desviospadrão dos Leq nos diferentes plantões e períodos de permanência do recém-nascido na sala de parto, isto é, antes do seu nascimento, durante a permanência do bebê na sala, e depois da saída da criança desse ambiente. Aplicando o teste de análise de variância (ANOVA) conclui-se que não há evidência de diferença dos Leq entre os diferentes plantões, antes e durante a permanência do recém- nascido na sala. Porém, constatou-se maior Leq médio significativo no plantão noturno quando comparados aos demais turnos, após o recém-nascido sair da sala de parto (Tabela 1).

Tabela 1 - Valores médios de Leq (dBA) por plantões e períodos de permanência do recém-nascido na sala de parto. São Paulo - SP, 2009

\begin{tabular}{cccccc}
\hline \multirow{2}{*}{ Plantão } & \multirow{2}{*}{ Estatística } & \multicolumn{2}{c}{ Leq (dBA) e permanência do recém-nascido } & \multirow{2}{*}{ Partos } \\
\cline { 3 - 5 } & & Antes & Durante & Depois & \\
\hline \multirow{2}{*}{ Manhã } & Média & 66,16 & 65,97 & 62,78 & 64,99 \\
& Desv.Pad & 1,80 & 2,59 & 2,16 & 1,44 \\
\multirow{2}{*}{ Tarde } & Média & 68,25 & 64,74 & 62,99 & 66,33 \\
& Desv.Pad & 5,62 & 2,23 & 2,41 & 4,02 \\
\multirow{2}{*}{ Noite } & Média & 66,73 & 67,28 & 66,72 & 67,44 \\
& Desv.Pad & 4,60 & 3,44 & 2,16 & 2,33 \\
& p-valor ANOVA & 0,585 & 0,169 & 0,004 & 0,305 \\
\hline
\end{tabular}

Para todos os partos pesquisados, pelo teste de ANOVA, verificou-se que há uma diferença do Leq médio no plantão noturno, quando comparado aos períodos da manhã e tarde $(\mathrm{p}=0,004)$.

Por outro lado, compararam-se os Leq médios nas três situações, durante todos os partos. Esses valores foram de 67,2 dBA, 65,8 dBA e 63,9 dBA antes do nascimento do bebê, durante sua permanência na sala, e após a saída desse ambiente, respectivamente. O teste de Friedmann comprovou pelo menos uma diferença significativa desses Leq com $\mathrm{p}$-valor=0,013.

A análise da relação entre o número de pessoas e o NPS registrado foi realizada por meio do coeficiente de correlação de Spearman. Concluiu-se que existe uma relação positiva e significativa entre o número de pessoas e o Leq registrado por parto $(\mathrm{r}=418 ; \mathrm{p}$-valor=0,033). Observou-se também que existe uma relação positiva e significativa $(\mathrm{r}=0,58)$; $p$-valor $=0,002$ ) entre o número de pessoas e os valores de Leq medidos no período estudado, ou seja, há um aumento do NPS, quanto maior o número de pessoas presentes na sala (Figura 1).

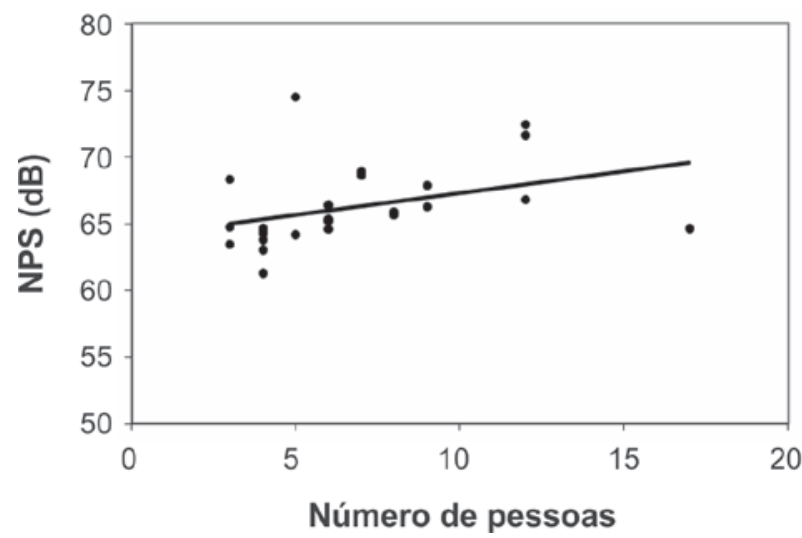

Figura 1 - Número de pessoas na sala de parto e a média dos níveis de pressão sonora durante os partos. São Paulo-SP, 2009

Comparando-se as médias de Leq entre os partos normais e cesáreas, antes do bebê nascer, identificou-se que o maior Leq foi 72,7 dBA nos partos normais e 75,7 dBA, nas cesáreas. Constatou-se essa diferença por meio do teste Mann Whitney $(\mathrm{p}=0,002)$. 
Em relação à identificação das fontes que produzem, calculou-se a frequência relativa, a partir das suas ocorrências, conforme o registro do pesquisador, por meio da observação nãoestruturada.

Antes de o bebê nascer, observou-se que as fontes de ruído mais frequentes foram a conversa da equipe dentro da sala $(88,0 \%)$, os diálogos estabelecidos entre os profissionais, no corredor do centro obstétrico $(25,0 \%)$, os alarmes dos equipamentos $(25,0 \%)$, arrastar de mesas, escadetes, bancos e equipamentos (21,0\%), abrir embalagens de materiais $(19,0 \%)$ e atrito de instrumentais cirúrgicos $(14,4 \%)$.

Durante a permanência do recém-nascido na sala, as principais fontes de ruído foram: conversa dos profissionais dentro da sala de parto $(94,0 \%)$, conversas no corredor $(17,0 \%)$, aspiração da cavidade abdominal da parturiente $(16,5 \%)$ e atrito de instrumentais cirúrgicos (11,0\%).

Após a saída do neonato da sala, as fontes mais frequentes de ruído constituíram-se na conversa dos profissionais no interior da sala $(85,0 \%)$, diálogos da equipe no corredor $(28,0 \%)$, atrito de instrumentais cirúrgicos e alarmes de equipamentos $(17,0 \%)$ e arrastar mesas, escadetes, bancos e equipamentos (13,0\%). Ressalta-se que, geralmente, na rotina do serviço, as portas das salas de parto permanecem abertas durante a realização dos procedimentos.

\section{DISCUSSÃO}

Todos os resultados encontrados são muito preocupantes, tanto para os recém-nascidos, quanto para as famílias e profissionais, uma vez que o menor valor de Leq médio foi $64,9 \mathrm{dBA}$.

Para a compreensão do impacto deste NPS para o recém-nascido, convém salientar que, dentro do útero, o bebê está exposto ao nível de ruído acima de $50 \mathrm{~dB}$, a baixas freqüências, com eventuais picos acima de $70 \mathrm{~dB},{ }^{15-17}$ mas a barreira constituída pela parede uterina e líquido amniótico, pode reduzir o ruído em até $35 \mathrm{~dB} \cdot{ }^{18}$ Ainda que a permanência mínima do neonato na sala tenha sido de sete minutos, evidencia-se que a recepção do bebê está inadequada, no que diz respeito ao seu acolhimento, podendo acarretar-lhe consequências fisiológicas e comportamentais no período de transição, que exige um grande esforço adaptativo. Ruídos altos e repentinos entre 70 a 75 dBA podem causar agitação e choro do bebê, resultando em aumento da pressão intracraniana, alterações das frequências cardíaca e respiratória, assim como diminuição da saturação de oxigênio transcutânea. Diante da fragilidade vascular e neuronal do cérebro do neonato, esses efeitos são potencialmente perigosos, porque podem causar alteração na perfusão do tecido cerebral. ${ }^{19}$

Considerando a questão comportamental, ressalta-se que em torno da primeira hora de vida após o nascimento, o recém-nascido apresenta o estado comportamental denominado de inatividade alerta, estado este no qual o bebê se mostra mais receptivo às interações com seus pais. Um ambiente acústico inadequado para o neonato na sala de parto poderá constituir-se em agressões sonoras que poderão levá-lo ao estresse, à irritabilidade ou ao desconforto, provocando o choro e, consequentemente, dificultar o encontro inicial entre pais e bebês, fundamental para o desenvolvimento do apego. ${ }^{20}$

Ansiedade e insegurança podem ser sentimentos comuns às gestantes durante quase toda a gravidez, por se tratar de uma nova situação e da necessidade da família assumir novos papéis. ${ }^{21}$ Portanto, um ambiente desconhecido e acusticamente inadequado em sala de parto, pode prolongar e até mesmo acentuar a instabilidade emocional da mulher.

Ainda, levando-se em consideração os altos NPSs obtidos nesse estudo, a permanência da equipe multiprofissional e de alunos, por períodos de seis a 12 horas, no centro obstétrico, poderão causar-lhes inúmeros efeitos deletérios. Entre esses, destacam-se a perda progressiva da audição, hipertensão arterial, aumento da liberação de catecolaminas, vasoconstrição, aumento da liberação do hormônio adrenocorticotrópico, cortisol, peristaltismo e tensão muscular, elevação de colesterol, distúrbio do sono e alterações no sistema imunológico, ${ }^{22,23}$ além de desencadear irritabilidade, distúrbios de humor, gerar fadiga e inibir a motivação para interagir com os clientes. ${ }^{24-28} \mathrm{O}$ ruído no ambiente de trabalho pode induzir o profissional à distração e ao consequente erro, ameaçando a segurança dos clientes.

Evidenciou-se que a conversação entre os profissionais, no interior da sala, foi a fonte de ruído mais frequente em todos os partos. Esses resultados assemelham-se aos encontrados em pesquisas realizadas em UTIN,,$^{29,30}$ indicando a importância do comportamento dos profissionais na determinação do perfil acústico das salas de partos estudadas. Ressalta-se que o ruído do corredor foi a segunda fonte mais frequente e, considerando 
que a portas das salas permaneceram abertas a maior parte do tempo, reforça-se a importância do fator comportamental da equipe.

\section{CONSIDERAÇÕES FINAIS}

Este estudo indica que o acolhimento do neonato e sua família na sala de parto está inadequado, considerando os elevados NPSs a que estão expostos.

Considerando que as fontes mais frequentes de ruído estão relacionadas ao comportamento dos profissionais e alunos, esse estudo aponta para a necessidade de realizar intervenções por meio de programas educativos a fim de minimizar o ruído e melhorar a assistência ao neonato e à família, e proporcionar melhores condições de trabalho da equipe, além de garantir a segurança do paciente.

Considerando ainda a importância dos primeiros momentos de vida para o desenvolvimento posterior do neonato, sugere-se o manejo adequado do ambiente, incorporando a perspectiva do cuidado desenvolvimental ao neonato nas salas de parto.

\section{AGRADECIMENTO}

À Fundação de Amparo à Pesquisa do Estado de São Paulo - FAPESP, pelo apoio para a realização da pesquisa (processo $n^{\circ}$ 0857294-8).

\section{REFERÊNCIAS}

1. Bremmer P, Byers JF, Kiehl E. Noise and the premature infant: physiological effects and practice implications. J Obstet Gynecol Neonatal Nurs. 2003 Jul-Aug; 32(4):447-54.

2. Schafer RM. A afinação do mundo: uma explanação pioneira pela história passada e pelo atual estado do mais negligenciado aspecto do nosso ambiente: a paisagem sonora. São Paulo (SP): UNESP; 2001.

3. Standley JM. A meta-analysis of the efficacy of music therapy for premature infants. J Pediat Nurs. 2002 Apr; 17(2):107-13.

4. Associação Brasileira de Normas Técnicas. NBR 10152. Níveis de ruídos para conforto acústico. Rio de Janeiro (RJ); 1987.

5. World Health Organization. Guidelines values. Guidelines for community noise page. 1999 [acesso 2003 Ago 18]. Disponível em: http:/ / www.who.int/ docstore/peh/noise/Commnoise4.htm

6. Ministério do Trabalho e Emprego (BR). NR-15. Atividades e operações insalubres (115.000-6) 08 Jun 1978. [acesso 2005 Abr 15]. Disponível em: http:/ /
www.mtb.gov.br/temas/SegSau/Legislacao/ Normas/Default.asp.

7. Ministério da Saúde (BR), Secretaria de Política de Saúde. Parto, aborto e puerpério: assistência humanizada à mulher. Brasília (DF): MS; 2001.

8. Graven SN. Sound and the developing infant in the NICU: conclusions and recommendations for care. J Perinatol. 2000 Dec; 20(8 Pt 2):S88-93.

9. Taylor HS, Klein N, Hack M.School age consequences of birth weight less than $750 \mathrm{~g}$ : a review and update. Dev Neuropsychol. 2000; 17(3):289-321.

10. Sampieri RH, Collado CF, Lucio PB. Metodologia de pesquisa. São Paulo (SP): Mc Graw Hill; 2006.

11. Associação Brasileira de Normas Técnicas. NBR 10151. Acústica - avaliação do ruído em áreas habitadas, visando o conforto da comunidade procedimento. Rio de Janeiro (RJ): ABNT; 2000.

12. Kakehashi TY, Pinheiro EM, Pizzarro G, Guilherme A. Nível de ruído em unidade de terapia intensiva neonatal. Acta Paul Enferm. 2007 Out-Dez; 20(4):404-9.

13. Ministério do Trabalho (BR). NR-15: Atividades e operações insalubres. Brasília (DF): Ministério do Trabalho; 1978 [acesso 2009 Nov 16]. Disponível em http://www.trabalho.gov.br/Empregador/ segsau/Legislacao/Portarias/1978/conteudo/ port_3214.asp

14. Kakehashi TY, Pinheiro EM. A observação em pesquisa qualitativa. In: Matheus MCC, Fustinoni SM. Pesquisa qualitativa em enfermagem. São Paulo (SP): Livraria médica paulista editora; 2006. p.106-7.

15. Abrams RM, Gerhardt KJ. The acoustic environment and physiological responses of the fetus. J Perinatol. 2000 Dec; 20(8 Pt 2):S31-6.

16. Gerhardt KJ, Abrams RM. Fetal exposures to sound and vibroacoustic stimulation. J Perinatol. 2000 Dec; 20(8 Pt 2):S21-30.

17. Gerhardt KJ, Pierson LL, Huang X, Abrams RM, Rarey KE. Effects of intense noise exposure on fetal sheep auditory brain stem response and inner ear histology. Ear Hear. 1999 Feb; 20(1):21-32.

18. Cruvinel FG, Pauletti CM. Formas de atendimento humanizado ao recém-nascido pré-termo ou de baixo peso na unidade de terapia intensiva neonatal: uma revisão. Cadernos de Pós-Graduação em Distúrbios do Desenvolvimento, 2009; 9(1):102-25.

19. Long JG, Lucey JF, Philip AGS. Noise and hypoxemia in the intensive care unit nursery. Pediatrics. 1980 Jan; 65(1):143-5.

20. Klaus MH, Klaus PH. O surpreendente recémnascido. Porto Alegre (RS): Artmed; 2001; 22-8.

21. Szer M, Steward R. Nove meses na vida da mulher. São Paulo (SP): Casa do Psicólogo; 1997.

22. Andren L, Hansson L, Eggertsen R, Hedner T, Kalberg BE. Circulatory effects of noise. Acta Med Scand. 1983; 213(1):31-5. 
23. Tomei F, Tomao E, Baccolo TP, Papaleo B, Alfi P. Vascular effects of noise. Angiology. 1992 Nov; 43(11):904-12.

24. Millar K, Steels MJ. Sustained peripheral vasoconstriction while working in continuous intensive noise. Aviat Space Environ Med. 1990 Aug; 61(8):695-8.

25. Melamed S, Bruhis S. The effects of chronic industrial noise exposure on urinary cortisol, fatigue and irritability: a controlled field experiment. J Occuped Environ Med. 1996 Mar; 38(3):252-6.

26. Willener P, Neiva P. Brief exposure to uncontrollable but not to controllable noise biases the retrieval of information from memory. Br J Chin Psychol. 1986 May; 25(Pt 2):93-100.
27. Pinheiro EM, Silva MJP, Angelo M, Ribeiro CA. The meaning of interaction between nursing professionals and newborns families in a hospital setting. Rev Latino-am Enfermagem. 2008 Nov-Dez; 16(6):1012-8.

28. Thomas KA, Martin PA. The acoustic environment of hospital nurseries: NICU sound environment and the potential problems for caregivers. J Perinatol. 2000; 20:93-8.

29. Chen HF, Chang YJ. Noise distribuction of an incubator with nebulizer at a neonatal intensive care unit in Southern Taiwan. J Nurs Res. 2001 Jun; 9(3):25-32.

30. Byers JF, Waugh WR, Lowman LB. Sound level exposure on high-risk infants in different environmental conditions. Neonatal Netw. 2006 Jan-Fev; 25(1):25-32. 